\title{
Spinal or Epidural Analgesia? Difference in Methods
}

(Rev Bras Anestesiol, 2010;60:5:484-494)

\section{Dear Editor,}

We read the paper published in the Journal with great interest on the comparative study of the analgesic role of bupivacaine (S75-R25) and ropivacaine in labor pain control ${ }^{1}$. The study gave original information regarding the analgesic effect of the newly mixed solution of bupivacaine containing $75 \%$ of the $\mathrm{S}$ isomer and $25 \%$ of the $\mathrm{R}$ isomer and ropivacaine, and the authors declared that these two drugs can provide good conditions for spinal anesthesia with small indices of adverse events. After careful reading of the whole methods of the study, we are confused about the study protocol. In the title and abstract of the paper, the authors used "spinal anesthesia", at first sight, to indicate that they administered these two drugs into subarachnoid space, but after following reading of the whole paper, we found the authors in fact performed continuous epidural analgesia, but not what they have demonstrated "spinal anesthesia".

Although spinal and epidural anesthesia all belong to regional anesthesia, there are big differences between these two techniques. Spinal anesthesia requires the injection of local anesthetics into subarachnoid space, but into peridural space when epidural anesthesia is performed. All theses two metho- ds produce contrasting effect on anesthesia or analgesia with different mechanisms ${ }^{2}$. As declared, the authors used "spinal anesthesia" in their study; this was a big error in definition of these two anesthetic methods. So we really do not know what anesthesia was used in their study. If such reply is not provided, the results of their paper will mislead readers and produce negative effects on the scientific research and clinical practice. Therefore, this paper should be withdrawn.

FuZhou Wang, MD Senior Lecturer, Research Consultant, ShanWu Feng, MD ShiQin Xu, MD QingSong Zhao, MD Staff Anesthesiologist YuSheng Liu, MD Lecturer, Staff Anesthesiologist XiaoFeng Shen, $M D$ Director Department of Anesthesiology and Critical Care Medicine, Nanjing Maternity and Child Health Care Hospital, Nanjing Medical University, China 


\section{REFERÊNCIAS / REFERENCES}

1. Nogueira CS, Lima LC, Paris VC et al. A comparative study between bupivacaine (S75-R25) and ropivacaine in spinal anesthesia for labor analgesia. Rev Bras Anestesiol, 2010;60:484-494.

2. Eltzschig HK, Lieberman ES, Camann WR. Regional anesthesia and analgesia for labor and delivery. N Engl J Med, 2003;348:319-332. 